Invest. 101:746-754.

13. Loetscher, M., Loetscher, P., Brass, N., Meese, E., and Moser, B. 1998. Lymphocyte-specific chemokine receptor CXCR3: regulation, chemokine binding and gene localization. Eur. J. Immunol. 28:3696-3705.

14. Schroder, K., Hertzog, P.J., Ravasi, T., and Hume, D.A. 2004. Interferon-gamma: an overview of sig- nals, mechanisms and functions. J. Leukoc. Biol. 75:163-189.

15. Raghu, G., et al. 2004. A placebo-controlled trial of interferon gamma- $1 \mathrm{~b}$ in patients with idiopathic pulmonary fibrosis. N. Engl. J. Med. 350:125-133. 16. Strieter, R.M., Starko, K.M., Enelow, R.I., Noth, I., and Valentine, V.G. 2004. Effects of interferon gamma-1b on biomarker expression in idiopathic pulmonary fibrosis patients. Am. J. Respir. Crit. Care Med. doi:10.1164/rccm.200312-1670OC

17. Ziesche, R., Hofbauer, E., Wittmann, K., Petkov, V., and Block, L.H. 1999. A preliminary study of long-term treatment with interferon gamma$1 \mathrm{~b}$ and low-dose prednisolone in patients with idiopathic pulmonary fibrosis. N. Engl. J. Med. 341:1264-1269.

\title{
Adaptive cellular immunity in aortic aneurysms: cause, consequence, or context?
}

\author{
John A. Curci'1,2 and Robert W. Thompson ${ }^{1,2,3}$ \\ ${ }^{1}$ Department of Surgery (Section of Vascular Surgery), ${ }^{2}$ Department of Radiology, and ${ }^{3}$ Department of Cell Biology and Physiology, \\ Washington University School of Medicine, St. Louis, Missouri, USA.
}

\begin{abstract}
Abdominal aortic aneurysms are common and life threatening. Although $\mathrm{CD}^{+} \mathrm{T}$ cells are abundant in aneurysm tissue, their role in disease progression remains unclear. A new study (see the related article beginning on page 300) shows that mouse aortic allografts placed in animals lacking IFN- $\gamma$ receptors develop a Th 2 inflammatory response with aortic aneurysms, whereas Th1 responses promote intimal hyperplasia. It is expected that these surprising findings will stimulate further efforts to clarify whether adaptive cellular immunity in aneurysm disease is detrimental or potentially beneficial.
\end{abstract}

The propensity of abdominal aortic aneurysms (AAAs) to rupture is their most important clinical feature, a consequence of increased hemodynamic stresses placed on the dilated wall, diminished tensile strength within the outer media and adventitia, and dynamic factors influencing the balance between matrix metabolism and repair. Surgical repair of AAAs greater than $5.5 \mathrm{~cm}$ in diameter is effective treatment, but repair of smaller aneurysms offers no survival advantage. Effective nonsurgical treatments to prevent aneurysm expansion would therefore be an enticing prospect for patients with small AAAs (1).

\section{Pathophysiology of AAAs}

Figure 1 presents a summary of pathophysiological events currently thought to contribute to aneurysmal degeneration, based on studies of human end-stage AAA tissues and several different experimental animal

Nonstandard abbreviations used: abdominal aortic aneurysm (AAA); IFN- $\gamma$ receptor deficient (GRKO); smooth muscle cell (SMC); tissue inhibitor of metalloproteinase (TIMP).

Conflict of interest: The authors have declared that no conflict of interest exists.

Citation for this article:

J. Clin. Invest. 114:168-171 (2004)

doi:10.1172/JCI200422309. models. Although the specific etiology is still unclear, aneurysms are probably initiated by aortic wall injury coupled with a series of epidemiological risk factors. Recruitment of leukocytes into the aortic media appears to be an early and pivotal event, likely prodation peptides (3). Mononuclear phagocyte infiltration is associated with production of proinflammatory cytokines (4), prostaglandin derivatives (5), and reactive oxygen species (6) as part of an innate inflammatory response. These macrophages are the principle source of MMPs (7), which can also be secreted by neutrophils, lymphocytes, and resident mesenchymal cells. Gelatinase B (MMP-9) has been extensively studied in human AAAs (8), but many other MMPs and endogenous tissue inhibitors of metalloproteinases (known as TIMPs) have also been described. Animal models of aortic aneurysm confirm that MMPs produced by chronic inflammatory cells are mediators of elastin and collagen degradation (9-11); moreover, the suppression of experimental aneurysms by MMP inhibitors has led to a promising therapeutic strategy (12). Other enzymes expressed in atherosclerosis and AAAs, particularly plasminogen activators and cathepsins, may also contribute to matrix proteolysis. moted by chemokines (2) and elastin degra-
Degradation of elastin and interstitial collagen initiates aortic dilatation and tortuosity, with changes in aortic wall geometry increasing cyclic strain and wall tension over a period of years. At later stages of disease, disorganized interstitial collagen is deposited within the media and adventitia, and collagen degradation becomes more prominent, further weakening the aortic wall. Although medial smooth muscle cells (SMCs) might otherwise promote structural repair in the damaged aorta, apoptosis and cellular senescence cause depletion of this cell population $(13,14)$.

\section{Adaptive immunity in aortic aneurysms}

In addition to macrophages, human AAAs demonstrate large numbers of $\mathrm{T}$ cells, B lymphocytes, plasma cells, and DCs within the outer media and adventitia $(15,16)$. AAA tissues also contain large amounts of immunoglobulin protein, and IgG extracted from human AAAs exhibits immunoreactivity with aortic wall matrix proteins (17). This suggests that a humoral (auto)immune response is a frequent occurrence in AAAs. Recent work has led to identification of several putative antigens that may be novel extracellular matrix proteins associated with large arteries (18).

The specificity of the immune response in AAAs is still unclear, as B lymphocytes derived from AAAs exhibit an unrestricted repertoire of immunoglobulin heavy chain genes (19), and $T$ cell receptor diversity reflects a polyclonal response (20). In a recent and comprehensive analysis, Ocana et al. (21) demonstrated that aneurysm-infiltrating lymphocytes consist of activated memory cells express- 

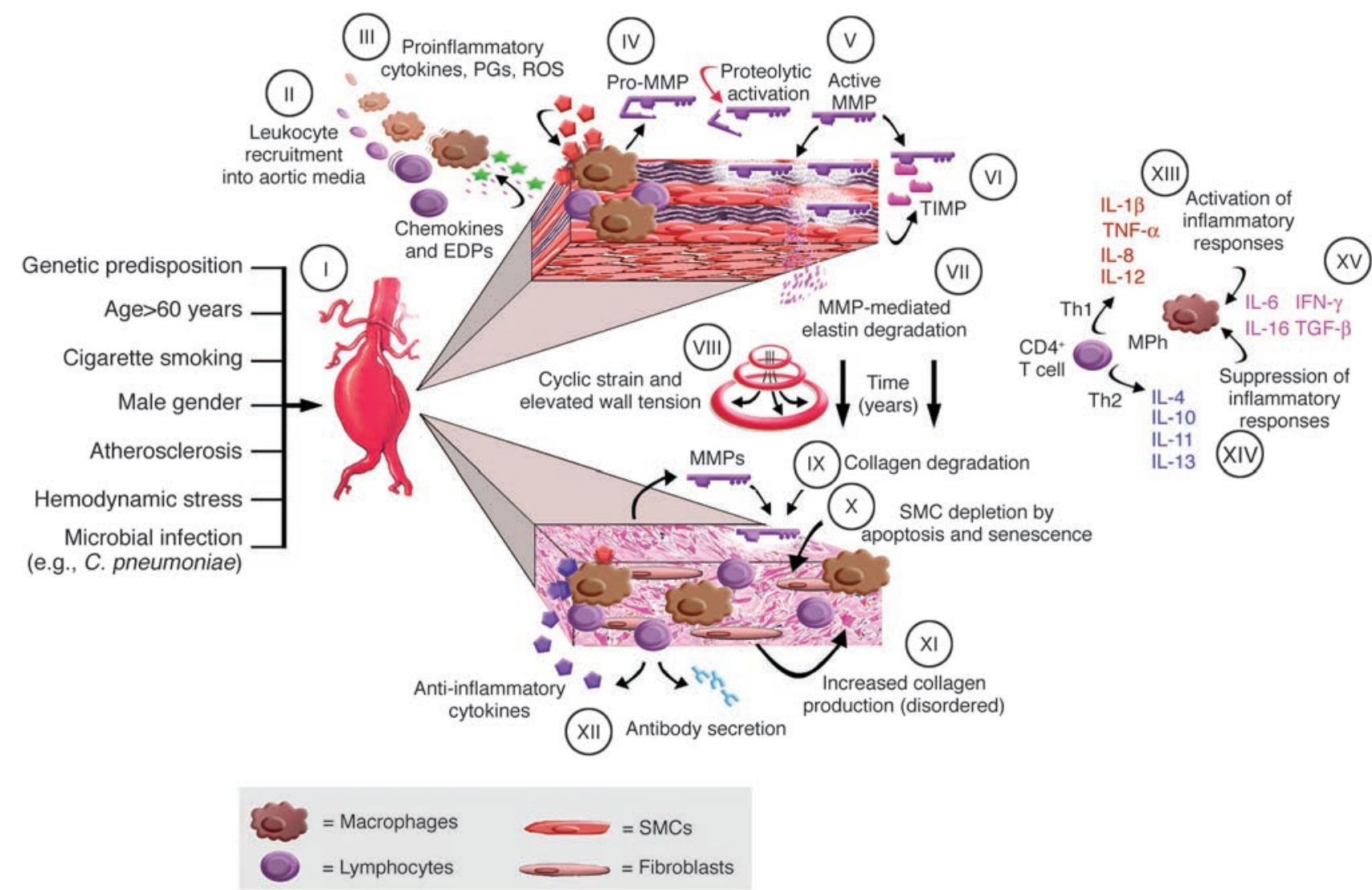

\section{Figure 1}

Pathophysiology of abdominal aortic aneurysms. Schematic diagram illustrating events thought to contribute to the development and progression of AAAs. Injury to the aortic wall, either as a consequence of or in association with known risk factors (I), leads to recruitment of leukocytes into the aortic media (II), macrophage activation, and production of proinflammatory molecules (III). Macrophages also produce proenzyme forms of MMPs (pro-MMPs) (IV), which are activated in the extracellular space (V). TIMPs may neutralize MMP activity (VI), but this appears insufficient to prevent degradation of structural matrix proteins (elastin and interstitial collagens) (VII). Over a period of years, elastin degradation, cyclic strain, and elevated wall tension bring about progressive aortic dilatation (VIII). Collagen degradation further weakens the aortic wall (IX); although medial SMCs and fibroblasts might promote structural repair, apoptosis and cellular senescence cause SMC depletion (X), and interstitial collagen appears disorganized (XI). Aneurysm tissues exhibit infiltration by T cells, B lymphocytes, plasma cells, and DCs and local deposition of immunoglobulins, reflecting a cellular and humoral immune response (XII). Understanding the adaptive cellular immune response in AAAs may reveal how different T cell subsets (i.e., Th1 versus Th2) interact with macrophages to promote or suppress aneurysmal degeneration, based on the local balance of proinflammatory (XIII) and anti-inflammatory (XIV) molecules. Some cytokines produced within aneurysm tissue, such as IL-6 and IFN- $\gamma$, may have dual and opposing functions depending on the specific context (XV). EDPs, elastin degradation peptides; MPh, macrophages; PGs, prostaglandins; ROS, reactive oxygen species.

ing costimulatory molecules. More specifically, $\mathrm{CD}^{+} \mathrm{T}$ cells predominated in AAA tissue, with expression of $\alpha \beta \mathrm{T}$ cell receptors, $T$ cell activation markers (CD69 and DR), a memory cell phenotype (CD45RO ${ }^{+} \mathrm{CD} 45 \mathrm{RA}^{-} \mathrm{CD} 62 \mathrm{~L}^{-}$), and a distinct pattern of cell surface molecules (including CD54, CD31, CD11a, CD29, CD44, CD95, and CD27). These results parallel observations in other chronic autoimmune/inflammatory disorders, supporting the existence of a cellular immune response in AAAs.

The triggers of adaptive immunity in AAAs are unknown, but there is often evidence of infection with Chlamydia pneumoniae in individuals with AAAs, and microbial infection might directly stimulate pathological immune responses in AAA tissue (22). Prior microbial infection might also target immune responses to aortic wall proteins through a process of molecular mimicry; alternatively, immune responses against aortic wall structural components may arise secondary to long-standing inflammation and connective tissue destruction, through proteolytic exposure of neoepitopes within matrix proteins.

The pathophysiologic implications of aortic wall cellular immune responses are an important focus for investigation, because cytokines produced by $\mathrm{T}$ cells (as well as direct interactions between T cells and activated macrophages) may have a substantial influence on macrophage production of MMPs and other matrixdegrading proteases. Proinflammatory cytokines associated with Th1 immune responses are elevated in the blood and aortic tissue of patients with AAAs (23), and circulating levels of IFN- $\gamma$ correlate with aneurysm expansion (24). Advanced human AAA tissues also express Th2-associated cytokines, particularly IL-4 and IL-10 (25). This is important given that IL-4 and IL-10 suppress human macrophage expression of MMP-9 (26), which suggests that Th2 immune responses might serve 
to restrain ongoing aneurysmal degeneration. In contrast, Schonbeck et al. (27) recently described that Th2 cytokines (IL-4, IL-5, and IL-10) were elevated to a greater extent in AAAs than in atheroma, where Th1 cytokines (IFN- $\gamma$, IL-2, IL-12, IL-15, and IL-18) predominated, and that IFN- $\gamma$ receptor expression was minimal in AAAs. This led the authors to propose that Th2 immune responses might actually direct atherosclerotic lesions toward aneurysm development rather than the formation of occlusive atheroma.

\section{Experimental studies}

Experimental studies are only beginning to help clarify the functional role of adaptive immunity in AAAs. For example, previous work from our laboratory indicated that mice lacking IL-10 developed larger aneurysms than did wild-type controls when subjected to elastase-induced AAAs, in association with more extensive degradation of elastin and collagen (28). Further studies have shown that larger aneurysms also occur in CD4-deficient and IL-4-deficient mice, which suggests the existence of a Th2 immune response that might otherwise suppress the extent of aneurysmal degeneration, whereas no significant effect on AAAs has been observed in mice with IFN- $\gamma$ deficiency (R.W. Thompson et al., unpublished results).

Using a calcium chloride-induced mouse model of AAAs, Baxter and colleagues (29) demonstrated that the absence of $\mathrm{CD}^{+} \mathrm{T}$ cells prevents the induction of experimental aneurysms. They also observed that administration of IFN- $\gamma$ reconstituted AAAs in CD4-deficient mice, that IFN- $\gamma$ deficient mice exhibited suppression of AAAs, and that the aneurysm-resistant phenotype was reversed by administration of wild-type splenocytes. These results lend strong support to the notion that Th1-type immune responses are detrimental in aneurysm disease. Based on this paradigm, one can envision future efforts to shift the cellular immune response from one dominated by Th1 cytokines to one favoring Th2 cytokines (perhaps by mucosal immunization strategies) in order to suppress aneurysmal degeneration.

Upon this background, Shimizu et al. (30) present an intriguing study in this issue of the JCI that examines the divergent functional roles of IFN- $\gamma$ and IL-4 in aortic pathology associated with a mouse model of arterial allotransplantation. The authors demonstrate that histocompat- ibility-mismatched aortas transplanted into IFN- $\gamma$ receptor-deficient (GRKO) recipients develop an immune response dominated by IL-4. These vessels subsequently develop severe inflammation, elastin degradation, increased expression of elastolytic MMPs (MMP-9 and MMP-12), and large aortic aneurysms. AAAs were not observed in allotransplants into wildtype recipients, which otherwise developed intimal hyperplasia. Furthermore, aneurysm development in GRKO allograft recipients was prevented by administration of anti-IL-4 blocking antibodies or by concomitant genetic deficiency in IL-4. Shimizu et al. conclude that IL-4 specifically mediates an inflammatory process leading to aneurysmal degeneration in this allograft model - a surprising and potentially important finding that challenges current concepts of how adaptive cellular immune responses might influence arterial disease.

The work by Shimizu et al. (30) has potentially valuable implications for the pathophysiology of allograft arteriopathy, intimal hyperplasia, arteritis, and AAAs and directly contradicts the paradigm that Th1 immune responses contribute to aneurysmal degeneration. There are several issues complicating interpretation of this study, however, and a number of questions that still need to be addressed. For example, the experimental model involved transplantation of histocompatibility-mismatched aortic tissues into genetically altered recipients. Although the authors note that $\mathrm{CD}^{+}$ $\mathrm{T}$ cells were rarely observed within the transplanted aortic wall, it seems unlikely that the allograft response can be overlooked in assessing the results, since MHC incompatibility would also affect $\mathrm{CD} 4^{+} \mathrm{T}$ cell responses. Secondly, it is apparent that after wild-type aortas were transplanted into GRKO recipients, resident cells within the graft were still able to respond to IFN- $\gamma$, while only the GRKO host (inflammatory) cells were unresponsive. This suggests that development of a Th1 response (as indicated by the large observed increase in IFN- $\gamma$ expression) might still have had an effect on cells within the aortic graft. It is not known if the activity of other Th1 cytokines might be enhanced in this experimental context, where IFN- $\gamma$ is unable to exert restraint on inflammatory cell function; indeed, previous studies in IFN- $\gamma$-deficient mice demonstrate a marked increase in inflammatory responses (31). A third issue that needs to be considered is the peculiar role played by IFN- $\gamma$ in maintaining the immunologic integrity of large vessels. Virgin and colleagues have demonstrated that herpesvirus infection of GRKO mice causes a severe and lethal panarteritis restricted to the large elastic arteries (32). In normal and GRKO mice there is persistent infection of aortic medial SMCs due to failure to clear infection from this location, as opposed to other organs or areas of the vessel wall (33). Persistence of disease is also accompanied by failure of $T$ cells and macrophages to enter the elastic media, suggesting that the aortic media is an immunoprivileged site normally maintained by IFN- $\gamma$. Loss of IFN- $\gamma$-mediated immune privilege within the aortic media may therefore have contributed to the development of aneurysms observed in GRKO recipients of aortic allografts (30). Thus, it is difficult to determine how aneurysms arising in this experimental model might be related to other animal models based on induction of nonspecific aortic wall inflammatory responses or, more importantly, how these findings might relate to human AAAs.

\section{Cause, consequence, or context?}

Based on the limited information accumulated to date, it is not yet known if adaptive cellular immune responses play a causative role in AAAs, or if they are simply a consequence of long-standing inflammation and connective tissue degradation. The bulk of evidence seems to suggest that elevated local production of Th1 cytokines likely serves to enhance macrophage expression of matrix-degrading proteinases and thereby accelerate aneurysmal degeneration, whereas Th2 cytokines appear to exert responses that would tend to suppress macrophage MMP production and limit disease progression. Conflicting experimental results may therefore simply reflect nuances attributable to different animal models of aneurysm disease and the complexities of context-specific inflammatory responses within the aortic wall. Some cytokines produced within aneurysm tissue clearly have dual and opposing functions, depending on the specific situation in which they are studied, and this variability may also extend to different models and stages of disease. For the present, better understanding of the contextual role of the adaptive cellular immune response in AAAs is needed. These efforts can be expected to reveal novel insights into how different $T$ cell subsets might interact with macrophages to promote or suppress aneu- 
rysmal degeneration, based on the local balance of proinflammatory and anti-inflammatory molecules, ultimately leading to clinically useful applications.

\section{Acknowledgments}

This work was supported by NIH grants HL56701, HL64332, and HL64333 (R.W. Thompson). John A. Curci is supported by a grant from the Barnes-Jewish Hospital Foundation and an American College of Surgeons Faculty Fellowship Award.

Address correspondence to: Robert W. Thompson, Section of Vascular Surgery, Washington University School of Medicine, 5101 Queeny Tower, One Barnes-Jewish Hospital Plaza, St. Louis, Missouri 63110, USA. Phone: (314) 362-7410; Fax: (314) 747-3548; E-mail: thompsonr@msnotes.wustl.edu.

1. Powell, J.T., and Brady, A.R. 2004. Detection, management, and prospects for the medical treatment of small abdominal aortic aneurysms. Arterioscler. Thromb. Vasc. Biol. 24:241-245.

2. Koch, A., et al. 1993. Enhanced production of the chemotactic cytokines interleukin-8 and monocyte chemoattractant protein-1 in human abdominal aortic aneurysms. Am. J. Pathol. 142:1423-1431.

3. Hance, K.A., Tataria, M., Ziporin, S.J., Lee, J.K., and Thompson, R.W. 2002. Monocyte chemotactic activity in human abdominal aortic aneurysms: role of elastin degradation peptides and the $67-\mathrm{kD}$ cell surface elastin receptor. J. Vasc. Surg. 35:254-261.

4. Newman, K.M., Jean-Claude, J., Li, H., Ramey, W.G., and Tilson, M.D. 1994. Cytokines that activate proteolysis are increased in abdominal aortic aneurysms. Circulation. 90:II224-II227.

5. Walton, L.J., et al. 1999. Inhibition of prostaglandin E2 synthesis in abdominal aortic aneurysms: implications for smooth muscle cell viability, inflammatory processes, and the expansion of abdominal aortic aneurysms. Circulation. 100:48-54.

6. Miller, F.J., Jr., et al. 2002. Oxidative stress in human abdominal aortic aneurysms: a potential mediator of aneurysmal remodeling. Arterioscler. Thromb. Vasc. Biol. 22:560-565.

7. Freestone, T., et al. 1995. Inflammation and matrix metalloproteinases in the enlarging abdominal aortic aneurysm. Arterioscler. Thromb. Vasc. Biol. 15:1145-1151

8. Thompson, R.W., et al. 1995. Production and localization of 92-kilodalton gelatinase in abdominal aortic aneurysms: An elastolytic metalloproteinase expressed by aneurysm-infiltrating macrophages. J. Clin. Invest. 96:318-326.

9. Pyo, R., et al. 2000. Targeted gene disruption of matrix metalloproteinase-9 (gelatinase B) suppresses development of experimental abdominal aortic aneurysms. J. Clin. Invest. 105:1641-1649.

10. Daugherty, A., Manning, M.W., and Cassis, L.A. 2000. Angiotensin II promotes atherosclerotic lesions and aneurysms in apolipoprotein E-deficient mice. J. Clin. Invest. 105:1605-1612.

11. Longo, G.M., et al. 2002. Matrix metalloproteinases 2 and 9 work in concert to produce aortic aneurysms. J. Clin. Invest. 110:625-632. doi:10.1172/ JCI200215334.

12. Mosorin, M., et al. 2001. Use of doxycycline to decrease the growth rate of abdominal aortic aneurysms: a randomized, double-blind, placebo-controlled pilot study. J. Vasc. Surg. 34:606-610.

13. Henderson, E.L., et al. 1999. Death of smooth muscle cells and expression of mediators of apoptosis by $\mathrm{T}$ lymphocytes in human abdominal aortic aneurysms. Circulation. 99:96-104.

14. Liao, S., Curci, J.A., Kelley, B., Sicard, G.A., and Thompson, R.W. 2000. Accelerated replicative senescence of medial smooth muscle cells derived from abdominal aortic aneurysms as compared to the adjacent inferior mesenteric artery. J. Surg. Res. 92:85-95.

15. Koch, A.E., et al. 1990. Human abdominal aortic aneurysms. Immunophenotypic analysis suggesting an immune-mediated response. Am. J. Pathol. 137:1199-1213.

16. Bobryshev, Y.V., and Lord, R.S. 2001. Vascular-associated lymphoid tissue (VALT) involvement in aortic aneurysm. Atherosclerosis. 154:15-21.

17. Gregory, A.K., et al. 1996. Features of autoimmunity in the abdominal aortic aneurysm. Arch. Surg. 131:85-88.

18. Hirose, H., Ozsvath, K.J., Xia, S., and Tilson, M.D. 1997. Molecular cloning of the complementary DNA for an additional member of the family of aortic aneurysm antigenic proteins. J. Vasc. Surg. 26:313-318.

19. Walton, L.J., Powell, J.T., and Parums, D.V. 1997. Unrestricted usage of immunoglobulin heavy chain genes in B cells infiltrating the wall of atherosclerotic abdominal aortic aneurysms. Atherosclerosis. 135:65-71.

20. Yen, H.C., Lee, F.Y., and Chau, L.Y. 1997. Analysis of the $\mathrm{T}$ cell receptor $\mathrm{V}$ beta repertoire in human aortic aneurysms. Atherosclerosis. 135:29-36.
21. Ocana, E., Bohorquez, J.C., Perez-Requena, J., Brieva, J.A., and Rodriguez, C. 2003. Characterisation of $\mathrm{T}$ and $\mathrm{B}$ lymphocytes infiltrating abdominal aortic aneurysms. Atherosclerosis. 170:39-48.

22. Halme, S., et al. 1999. Chlamydia pneumoniae reactive T lymphocytes in the walls of abdominal aortic aneurysms. Eur. J. Clin. Invest. 29:546-552.

23. Szekanecz, Z., Shah, M.R., Pearce, W.H., and Koch, A.E. 1994. Human atherosclerotic abdominal aortic aneurysms produce interleukin (IL)- 6 and interferon-gamma but not IL-2 and IL-4: the possible role for IL- 6 and interferon-gamma in vascular inflammation. Agents Actions. 42:159-162.

24. Juvonen, J., et al. 1997. Elevated circulating levels of inflammatory cytokines in patients with abdominal aortic aneurysm. Arterioscler. Thromb. Vasc. Biol. 17:2843-2847.

25. Davis, V.A., et al. 2001. Cytokine pattern in aneurysmal and occlusive disease of the aorta. J. Surg. Res. 101:152-156.

26. Lacraz, S., Nicod, L.P., Chicheportiche, R., Welgus, H.G., and Dayer, J.M. 1995. IL-10 inhibits metalloproteinase and stimulates TIMP-1 production in human mononuclear phagocytes. J. Clin. Invest. 96:2304-2310.

27. Schonbeck, U., Sukhova, G.K., Gerdes, N., and Libby, P. 2002. T(H)2 predominant immune responses prevail in human abdominal aortic aneurysm. Am. J. Pathol. 161:499-506.

28. Geraghty, P.J., Starcher, B.C., Borhani, M., and Thompson, R.W. 2001. Interleukin-10 deficiency potentiates aortic collagen and elastin degradation in elastase-induced murine aortic aneurysm formation. Arterioscler. Thromb. Vasc. Biol. 21:676. (Abstr.)

29. Xiong, W., Zhao, Y., Prall, A., Greiner, T.C., and Baxter, B.T. 2004. Key roles of CD4(+) T cells and IFN-gamma in the development of abdominal aortic aneurysms in a murine model. J. Immunol. 172:2607-2612

30. Shimizu, K., Shichiri, M., Libby, P., Lee, R.T., and Mitchell, R.N. 2004. Th2-predominant inflammation and blockade of IFN- $\gamma$ signaling induce aneurysms in allografted aortas. J. Clin. Invest. 114:300-308. doi:10.1172/JCI200419855.

31. Dalton, D.K., et al. 1993. Multiple defects of immune cell function in mice with disrupted interferon-gamma genes. Science. 259:1739-1742.

32. Weck, K.E., et al. 1997. Murine gamma-herpesvirus 68 causes severe large-vessel arteritis in mice lacking interferon-gamma responsiveness: a new model for virus-induced vascular disease. Nat. Med. 3:1346-1353.

33. Dal Canto, A.J., Swanson, P.E., O'Guin, A.K., Speck, S.H., and Virgin, H.W. 2001. IFN-gamma action in the media of the great elastic arteries, a novel immunoprivileged site. J. Clin. Invest. 107:R15-R22. 